\title{
HIV Infection and Cancer: Multi-Institutional Collaboration is the Answer Jorge J. Castillo*
}

The Warren Alpert Medical School of Brown University, Division of Hematology and Oncology, Rhode Island Hospital/ The Miriam Hospital, Providence, RI, USA

An increased risk of cancers was identified early in the HIV/AIDS epidemic. It was in the summer of 1981 that a series of young homosexual men in New York and California who developed a rare malignancy known as Kaposi Sarcoma (KS) was presented [1], coinciding with another series of patients who were diagnosed with an atypical pneumonia caused by the then called Pneumocystis carinii, now known as $P$. jirovecci [2]. In the following years, although atypical infections would be associated with the gross of the morbidity and mortality experienced by HIV-infected individuals, the spectrum of cancers associated to HIV infection grew to include aggressive B-cell NonHodgkin Lymphoma (NHL) and Invasive Cervical Carcinoma (ICC). These three malignancies, KS, NHL and ICC, were then considered AIDS-Defining Cancers (ADCs) due to the relationship seen between the degree of immunosuppression and the incidence of these cancers. ADCs would comprise $>95 \%$ of all the cancer cases detected in HIVpositive patients with KS being by far the most common cancer seen in patients with HIV before the advent of Highly Active Antiretroviral Therapy (HAART). The often poor immunological and performance status of patients infected with HIV would make the treatment of these cancers with chemotherapy or radiotherapy rather difficult, plagued with infectious complications and high rates of mortality.

In recent years (post-HAART era), several changes in the HIV-cancer relationship have been observed. First, due to a relatively higher impact on opportunistic infections, cancer has become the leading cause of morbidity and mortality among HIV-infected individuals in the United States [3]. Second, the profile of the cancers diagnosed in HIV-positive patients has changed. The absolute incidence of KS and aggressive NHL has decreased but to a greater extent in the former, allowing NHL to become the most common malignancy and the first cause of cancerrelated mortality among HIV-infected individuals [4]. Third, the profile of aggressive NHL has also been modified. For example, the incidence of primary brain lymphoma and primary effusion lymphoma has decreased significantly since the advent of HAART. Fourth, now that HIV-infected individuals are living longer, the risk of other malignancies, not precisely associated with immunosuppression, is on the rise. Such cancers are known as Non-AIDS-Defining Cancers (NADCs). In recent population-based studies, NADCs comprise up to $50 \%$ of all the cases of cancers seen in patients with HIV/AIDS, showing and elevated risk of cancers such as Hodgkin Lymphoma (HL), lung, liver, head and neck, and anal cancer $[5,6]$. On the other hand, the risk of common cancers such as colon, breast and prostate do not appear increased in patients with HIV for reasons yet to be defined. Finally, with the improvement on the immunological and physical status of patients with HIV due to HAART, there has been a shift in the paradigm of the treatment of HIV-infected individuals who develop a malignancy. In many cases, HIV-positive patients with cancers nowadays can and will be treated following regimens similar to immunocompetent patients in dosage and intensity, although the use of growth factors and antibiotic prophylaxis is mandatory to decrease potentially devastating chemotherapy-related adverse events.

Despite these advances, the management of cancers in the context of HIV/AIDS remains challenging with several unanswered questions. One of the most challenging aspects is the potential interaction that HAART drugs such as Non-Nucleoside Reverse Transcriptase
Inhibitors (NNRTIs) or Protease Inhibitors (PIs) may have when used in combination with chemotherapeutics as relatively very little data has emerged in recent years. The main mechanism for drug metabolism is through the cytochrome P450 (CYP450) pathway. Many chemotherapeutic agents, NNRTIs and PIs are either inducers and/or inhibitors of CYP450; hence, one patient could be potentially receiving concurrently up to 4-5 medications that could induce or inhibit CYP450. A second mechanism of interaction is played through interference with the function of P-glycoprotein (P-gp). P-gp belongs to a family of transporters of molecules across cellular membranes. More specifically, P-gp works as an efflux pump for several compounds, including HIV and chemotherapy drugs decreasing or increasing the intracellular concentrations of such drugs. These interactions could function in two ways and could increase the bioavailability of some promoting accumulation and toxicity, or could decrease the concentration of others decreasing efficacy [7]. In a few studies, there have been attempts on stopping HAART during intensive anti-lymphoma regimens with reasonably good results [8]. But, on the other hand, several studies support the premise that HAART and chemotherapy should be administered together for better results, as either an immunological or a virological response to HAART appears associated with increased rates of response to chemotherapy and survival [9].

The treatment of cancer patients with HIV/AIDS is far from standardized; however, with a better understanding on the biology and pharmacology of HIV-associated malignancies and their therapy, few recommendations could be made. First, whenever possible, patients with HIV/AIDS and cancer should be treated as immunocompetent, mainly in patients with CD4+ counts higher than $200 / \mu \mathrm{L}$ and undetectable viral loads. It is of most importance to recognize that cancers such as aggressive NHL, HL and anal cancer can be cured, and patients with HIV/AIDS should be treated with this goal in mind. Second, HAART should be given concurrently to chemotherapy unless specific adverse events occur necessitating dose reductions or delays. This is mainly advisable for patients who are HAART-naïve and have low CD4+ counts. The benefits of the combination of HAART and chemotherapy have been mainly reported in patients with lymphomas and KS. Third, a more liberal use of supportive therapy, such as granulocyte-stimulating growth factors and antibiotic prophylaxis may translate into fewer infectious adverse events, either associated with neutropenic episodes or opportunistic infections. Finally, patients with HIV/AIDS and cancer should be treated by HIV oncologists in setting

${ }^{*}$ Corresponding author: Jorge J. Castillo, MD, The Warren Alpert Medical Schoo of Brown University, Division of Hematology and Oncology, Rhode Island Hospital/ The Miriam Hospital, 164 Summit Ave, Providence, RI 02906, USA, Tel: 4017937151; Fax: 401-7937132; E-mail: jcastillo@lifespan.org

Received April 21, 2012; Accepted April 24, 2012; Published April 28, 2012

Citation: Castillo JJ (2012) HIV Infection and Cancer: Multi-Institutional Collaboration is the Answer. J AIDS Clinic Res 3:e105. doi:10.4172/2155-6113.1000e105

Copyright: (c) 2012 Castillo JJ, et al. This is an open-access article distributed under the terms of the Creative Commons Attribution License, which permits unrestricted use, distribution, and reproduction in any medium, provided the original author and source are credited. 
Citation: Castillo JJ (2012) HIV Infection and Cancer: Multi-Institutional Collaboration is the Answer. J AIDS Clinic Res 3:e105. doi:10.4172/21556113.1000 e105

in which they could benefit from clinical trials designed specifically for such population.

Additional research is needed to further delineate the interactions between HIV medications and chemotherapy drugs, and to identify more effective therapies, which should results in increased cure rates or, in most cases, in an improved quality of life. Many of the published studies on the biology, pathophysiology or therapy of HIV-associated malignancies are single-center experiences or small prospective studies with very few randomized controlled trials. For this purpose, multiinstitutional collaboration is mandatory. In the United States, the AIDS Malignancy Consortium (AMC) is a clinical trials group supported by the National Cancer Institute specifically focused on performing clinical and laboratory studies in HIV/AIDS patients with cancer (For more information, please see http://pub.emmes.com/study/amc/ public/index.htm). The AMC is composed by over 35 clinical sites in the United States, Asia, Africa and Latin America, and has working groups focused on the treatment of lymphoma, KS, HPV-associated malignancies and NADCs. Such efforts are desperately needed in order to investigate the different aspects of HIV-associated malignancies therapy.

\section{References}

1. Centers for Disease Control (CDC) (1981) Kaposi's sarcoma and Pneumocystis pneumonia among homosexual men--New York City and California. MMWR Morb Mortal Wkly Rep 30: 305-308.

2. Centers for Disease Control (CDC) (1981) Pneumocystis pneumonia--Los Angeles. MMWR Morb Mortal Wkly Rep 30: 250-252.

3. Antiretroviral Therapy Cohort Collaboration (2010) Causes of death in HIV-1 infected patients treated with antiretroviral therapy, 1996-2006: collaborative analysis of 13 HIV cohort studies. Clin Infect Dis 50: 1387-1396.

4. Simard EP, Engels EA (2010) Cancer as a cause of death among people with AIDS in the United States. Clin Infect Dis 51: 957-962.

5. Crum-Cianflone N, Hullsiek KH, Marconi V, Weintrob A, Ganesan A, et al. (2009) Trends in the incidence of cancers among HIV-infected persons and the impact of antiretroviral therapy: a 20-year cohort study. AIDS 23: 41-50.

6. Powles T, Robinson D, Stebbing J, Shamash J, Nelson M, et al. (2009) Highly active antiretroviral therapy and the incidence of non-AIDS-defining cancers in people with HIV infection. J Clin Oncol 27: 884-890.

7. Spano JP, Costagliola D, Katlama C, Mounier N, Oksenhendler E, et al. (2008) AIDS-related malignancies: state of the art and therapeutic challenges. $\mathrm{J}$ Clin Oncol 26: 4834-4842.

8. Little RF, Pittaluga S, Grant N, Steinberg SM, Kavlick MF, et al. (2003) Highly effective treatment of acquired immunodeficiency syndrome-related lymphoma with dose-adjusted $\mathrm{EPOCH}$ : impact of antiretroviral therapy suspension and tumor biology. Blood 101: 4653-4659.

9. Hoffmann C, Wolf E, Fatkenheuer G, Buhk T, Stoehr A, et al. (2003) Response to highly active antiretroviral therapy strongly predicts outcome in patients with AIDS-related lymphoma. AIDS 17: 1521-1529. 